\title{
Pulsatilla decoction alleviates colitis by enhancing autophagy and regulating PI3K-Akt-mTORC1 signaling pathway
}

\author{
XUEWEI WANG ${ }^{*}$, LIJUN XU* ${ }^{*}$ TAO WANG, JIAN XU, FUGANG FAN, YU ZHANG, JINPIN WANG and QIN CAO \\ Division of Gastroenterology, Putuo Hospital, Shanghai University of Traditional Chinese Medicine, \\ Shanghai 200062, P.R. China
}

Received July 28, 2020; Accepted April 7, 2021

DOI: $10.3892 / \mathrm{mmr} .2022 .12624$

\begin{abstract}
The aim of the present study was to investigate the therapeutic effect of Pulsatilla decoction (PD) on ulcerative colitis (UC) and to elucidate its potential molecular mechanisms. C57BL/6 mice expressing natural killer (NK)1.1 were used as experimental animals in the present study and a model of oxazolone-induced colitis was established. Mice were randomly divided into the following five groups: i) PD group; ii) oxazolone-induced colitis group; iii) IL-13 intervention group; iv) 5-aminosalicylic acid positive control group; and v) negative control group (equal volume saline gavage). A total of 10 animals were used in each group. The effects of PD on UC and the association between this regimen and the PI3K-Akt-mTORC1 signaling pathway were evaluated by disease activity index (DAI), hematoxylin and eosin staining, reverse transcription-quantitative PCR (RT-qPCR), immunofluorescence assay, ELISA and western blotting. The UC models were successfully established by injecting oxazolone gavage solution. Clinical colitis evaluation and histological examination revealed that PD reduced the DAI values in oxazolone-induced colitis in mice and the degree of infiltration in NK1.1 cells. PD significantly reduced the secretion of IL-13, as determined using an ELISA. In addition, western blotting and RT-qPCR analyses demonstrated that Beclin1 and LC3II/I expression levels were downregulated following treatment of the mice with PD. In addition, PD not only partially restored alterations in the expression of tight junction proteins in the colon tissues, but also suppressed the activation of the PI3K-Akt-mTORC1 signaling pathway. The data indicated that this regimen could alleviate oxazolone-induced UC in mice, which could significantly reduce tissue inflammation
\end{abstract}

Correspondence to: Dr Qin Cao, Division of Gastroenterology, Putuo Hospital, Shanghai University of Traditional Chinese Medicine, 164 Lanxi Road, Shanghai 200062, P.R. China

E-mail: caoqin434@126.com

${ }^{*}$ Contributed equally

Key words: Pulsatilla decoction, ulcerative colitis, autophagy, PI3K-Akt-mTORC1 and autophagy. The mechanism of action was associated with the PI3K-Akt-mTORC1 signaling pathway.

\section{Introduction}

Ulcerative colitis (UC) is a chronic and idiopathic inflammatory disease of the colon, which is characterized by abdominal pain and mucopurulent bloody stools (1). This digestive system disease seriously affects patients' ability to work and their quality of life (2). UC can lead to colon cancer following long-term recurrence $(1,2)$. UC is a chronic non-specific inflammatory disease of the colon and rectum with unclear etiology, which is confined to the large intestinal mucosa and submucosa (3). The lesions mostly occur in the sigmoid and rectum but can also extend to the descending colon and even to the entire colon (3).

The majority of patients with UC have a mild disease that is mainly treated with 5-aminosalicylic acid (5-ASA), whereas patients with severe disease require hormone-induced remission $(4,5)$. It can change the transcription of inflammatory genes, and play anti-inflammatory and immunosuppressive roles, therefore it is the preferred therapeutic drug for patients with severe UC (4). However, in some patients, the oral drugs reach the distal colon, i.e., the second colon, or even part of the descending colon, at a low concentration, which makes it difficult to exert the efficacy and this is the site where UC is most likely to occur (5). Suppositories can only act on the rectal mucosa, so the inflammation of the distal colonic mucosa is often prolonged $(1,2)$. The drugs used often cause different degrees of bone marrow suppression and immunosuppression, leading to leukopenia (6). They can also induce tuberculosis and the formation of tumors (7). As a result, research exploring more effective treatments for UC is gaining considerable interest.

In recent years, herbal medicine has been characterized as one of the most common alternative and complementary therapeutic modalities that has been shown to be effective in treating UC (8). The biological activity of herbal medicine has been explored in terms of basic research and in terms of its clinical application $(6,7)$. It is of note that the combination of Chinese and Western medicine has been shown to be more effective than single conventional treatment for $\operatorname{UC}(8,9)$. The most famous Traditional Chinese medicine (TCM) used is Pulsatilla decoction (PD) (10). The successful application 
of PD suggests that herbal medicine may be a promising alternative therapy for the treatment of patients with UC (9).

UC mucosal immune inflammation is characterized by the accumulation of antigen-presenting cells, such as dendritic cells or the MHC-I-like molecule CD11d cells, which are present on the surface of colon epithelial cells (10). A previous study demonstrated that UC is associated with the presence of non-invasive (type II) natural killer (NK)T cells, which produce IL-13 and mediate cytotoxicity of epithelial cells (11). NKT cells secreting IL-13 is responsible for the primary inflammation caused in UC, and is a key molecular mediator that can induce colon epithelial cell apoptosis, inhibit the expression of intercellular tight junction proteins and prevent the reformation of epithelial cells, leading to epithelial barrier damage and causing intestinal bacteria translocation and further activation of macrophages, neutrophils, eosinophils, NKT and T cells (12). Therefore, the secretion of IL-13 by NKT cells is a key initiating event in UC.

PD is derived from Zhang Zhongjing's 'Shang Han Lun' and consists of four herbs, including radix pulsatillae (11), cortex fraxini, cortex phellodendri and rhizoma coptidis, which are effective in adjusting homeostasis and can induce anti-fungal effects in vitro (12). The nuclear transcription factor (13), NF- $\mathrm{NB}$, is important in monitoring the development and progression of inflammatory bowel diseases by regulating the expression levels of a wide range of pro-inflammatory cytokines (13). Clinical studies have reported that PD significantly suppresses the activation of the NF- $\kappa \mathrm{B}$ signaling pathway and cytokine expression, while it modulates the imbalance in the expression levels of the proinflammatory factor TNF- $\alpha$ and the anti-inflammatory factor IL-10 (14-16). In a previous study, an oxazolone-induced colitis model was established in BALB/c mice, which demonstrated typical symptoms of UC, such as diarrhea, hematochezia and weight loss, apparent hyperemia, edema and exudation in the diseased colon, pathological changes of chronic inflammation in the mucosa and a significant increase in the expression levels of the characteristic UC cytokines IL-13 (14,15). In the present study, the therapeutic effects of PD were investigated in an oxazolone-induced colitis model and its underlying molecular mechanism was elucidated.

\section{Materials and methods}

Establishment of a C57BL/6 murine model of oxazoloneinduced colitis. A total of 50 male C57BL/6 mice (age, 8 weeks; weight, $20 \mathrm{~g}$; SPF grade) expressing natural killer (NK)1.1 were provided by Shanghai SLAC Laboratory Animal Co., Ltd., and housed in a specific-pathogen-free environment under normal conditions of humidity $(50 \pm 5 \%)$ and temperature $\left(25 \pm 2^{\circ} \mathrm{C}\right)$ with a 12 -h light/dark cycle and access to food and water ad libitum. The mucosal immune response of $\mathrm{UC}$ is a Th2 lymphocytic-like response mediated by NKT cells. Therefore, NK1.1-expressing mice were used as the research subjects. Subsequently, on day 1 , the mice were anesthetized with $35-40 \mathrm{mg} / \mathrm{kg}$ pentobarbital sodium intraperitoneally and shaved on the back between the shoulders, subsequently a $2 \times 2 \mathrm{~cm}$ area of skin was exposed, which was treated with $200 \mu \mathrm{l}$ sensitizing solution (Sigma-Aldrich; Merck KGaA). On the day 2, the animals developed an allergic reaction. On day 5, fasting was initiated. On day 6 , following pentobarbital sodium anesthesia, a 3.5 F hose, lubricated with sterile paraffin oil (Sangon Biotech Co., Ltd.) was slowly inserted into the anus of the mouse (distance, $4 \mathrm{~cm}$ ). A total of $150 \mu \mathrm{l}$ oxazolone gavage solution (Sigma-Aldrich; Merck $\mathrm{KGaA}$ ) was slowly injected and the catheter was removed. The head of the animal was kept down in a vertical position for $60 \mathrm{sec}$ in order to leave the oxazolone solution in the intestinal lumen. All protocols were reviewed and approved by the ethics committee of Shanghai University of Traditional Chinese Medicine (approval no. 0015; Shanghai, China).

Preparation and intragastric administration of water-soluble extracts of $P D$. PD (16) was made up of $30 \mathrm{~g}$ radix pulsatillae, $9 \mathrm{~g}$ cortex fraxini, $5 \mathrm{~g}$ cortex phellodendri and $12 \mathrm{~g}$ rhizoma coptidis (all purchased from Longhua Hospital Shanghai University of Traditional Chinese Medicine; Shanghai, China). To obtain the water-soluble extracts, the samples were extracted using ethanol, and then concentrated, dried and powdered (17). The samples extracted by alcohol were autoclaved and stored at $4^{\circ} \mathrm{C}$. The mice were randomly divided into the following five groups, with 10 mice in each group: i) PD group; ii) oxazolone-induced colitis group; iii) IL-13 (1 mg/kg body weight) intervention using anti IL-13 (PeproTech; cat. no. 500-P178) group; iv) 5-ASA (cat. no. 461814-25G; Sigma-Aldrich; Merck $\mathrm{KGaA} ; 20 \mathrm{mg} / \mathrm{g}$ body weight) positive control group; and v) negative control group (equal volume saline gavage). The PD extracts ( $20 \mathrm{mg} / \mathrm{g}$ body weight) were administered intragastrically to the oxazolone-induced colitis mice once a day for 7 days.

Assessment of clinical colitis in mice. The disease activity index (DAI) was used to evaluate the grade and extent of intestinal inflammation according to an established index system (18). During the treatment, the body weight, stool characteristics and the degree of bloody stool were recorded daily and were subsequently assessed using a DAI scoring system. The body weight change in each mouse was calculated using the following formula: DAI $=[$ (percent weight loss + diarrheal stool score + bloody stool score)]/3. The scores ranged from 0 to 4 , including the following three parameters: Weight loss, stool consistency and rectal bleeding.

Reverse transcription-quantitative PCR (RT-qPCR). RT-qPCR was used to evaluate mRNA expression in different colon tissue groups. The extraction of total RNA was performed using TRIzol ${ }^{\circledR}$ reagent (Invitrogen; Thermo Fisher Scientific, Inc.) according to the manufacturer's instruction. Next, purified RNA was subjected to RT using the PrimeScript RT reagent kit (Takara Bio, Inc.). RT-qPCR was performed using SYBR Green qPCR Master Mix (Takara Bio, Inc.) as follows: Initial degeneration at $95^{\circ} \mathrm{C}$ for $30 \mathrm{sec}$, followed by 40 cycles of $95^{\circ} \mathrm{C}$ for $5 \mathrm{sec}$ and $60^{\circ} \mathrm{C}$ for $30-60 \mathrm{sec}$. The relative expression of mRNA (normalized against actin) was calculated by the $2^{-\Delta \Delta \mathrm{Cq}}$ method (19).

The following primers were used for each target gene: Beclin-1 forward, 5'-CCCAGCCAGGATGATGTCTAC-3' and reverse, 5'-AGGTCTCCAGTGACCTTGAGT-3'; LC3B forward, 5'-CCGTAGTTCGCTGTACGAGG-3' and reverse, 5'-AACTCACGTCGGATGTCCAG-3'; PI3K forward, 
A
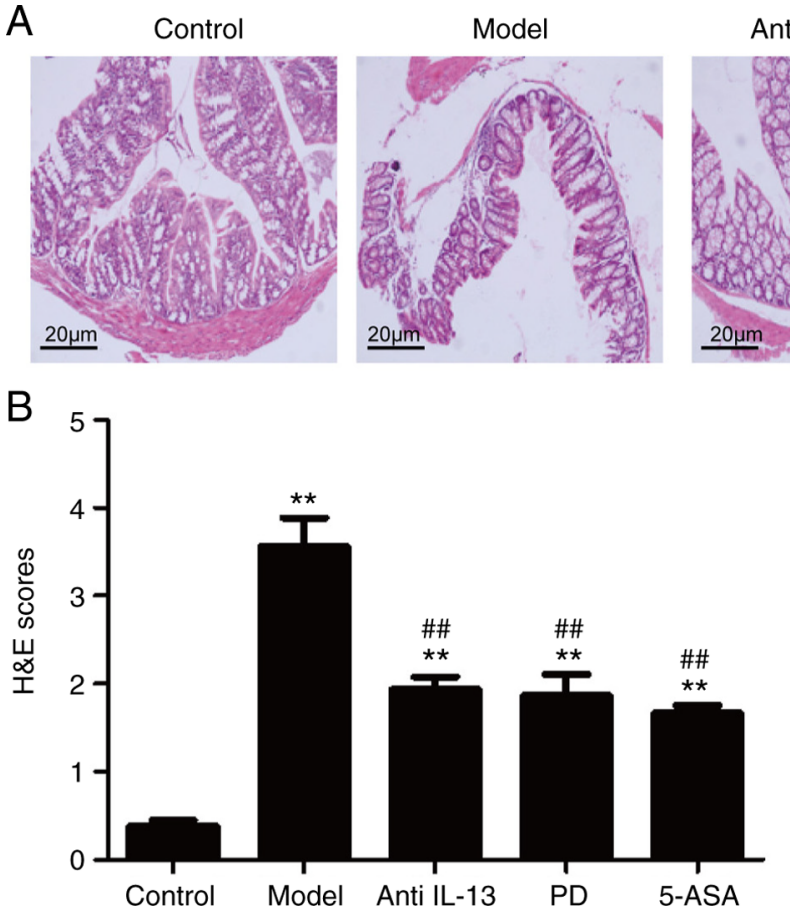

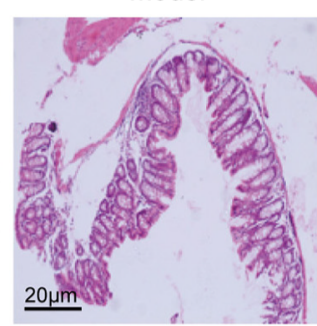

Anti IL-13

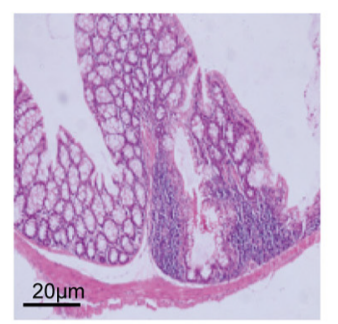

PD

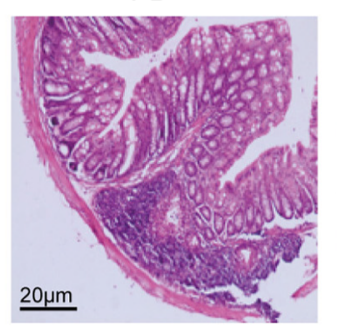

5-ASA

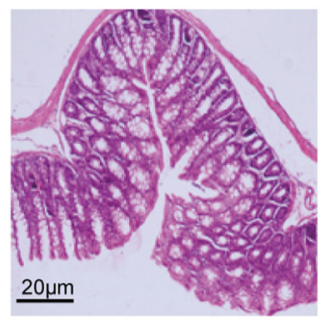

\section{C}

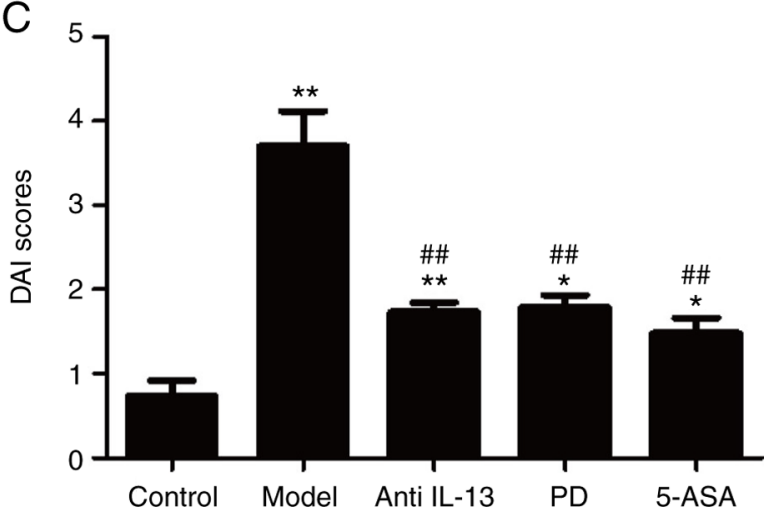

Figure 1. PD alleviates the symptoms of ulcerative colitis in mice. (A) Representative images from H\&E-stained sections of the colon derived from different groups of mice. Magnification, x200. (B) H\&E and (C) DAI scores of oxazolone-induced colitis mice were measured following oral administration of PD, anti-IL-13 intervention or 5-ASA for 7 days. The data are representative of three independent experiments and are expressed as the mean \pm standard deviation. ${ }^{*} \mathrm{P}<0.05,{ }^{* *} \mathrm{P}<0.01$ vs. normal control group; ${ }^{\# \#} \mathrm{P}<0.01$ vs. model control group. PD, Pulsatilla decoction; H\&E, hematoxylin and eosin; DAI, disease activity index; 5-ASA, 5-aminosalicylic acid.

5'-CTGGAATGTGTGGCTGGAGT-3' and reverse, 5'-AGG AGGAAGCGGTGGTCTAT-3'; Akt forward, 5'-ATGAAC GACGTAGCCATTGTG-3' and reverse, 5'-TTGTAGCCA ATAAAGGTGCCAT-3'; mTORC1 forward, 5'-ATCGTGCTG TTGGGTGAGAG-3' and reverse, 5'-TGGATCTCCAGCTCT CCGAA-3'; autophagy-related protein 13 (ATG13) forward, 5'-TGGACCATCAACCGGAAACT-3' and reverse, 5'-CAA GGGTATGCAGCTGTCCA-3'; zona occludens protein 1 (ZO-1) forward, 5'-CATGGCCGGGAATGATGAGA-3' and reverse, 5'-TGAAACTCTTGCCTCGTCCG-3'; claudin-2 forward, 5'-GATCGGCTCCATCGTCAGC-3' and reverse, 5'-TGTTGGGTAAGAGGTTGTTTTCC-3'; and GAPDH forward, 5'-AGGTCGGTGTGAACGGATTTG-3' and reverse, 5'-TGTAGACCATGTAGTTGAGGTCA-3'.

ELISA. ELISA kits (cat. no. ab219634; Abcam) were used to detect the amount of secretory IL-13 in the serum and the expression of myeloperoxidase (MPO; cat. no. PA5-16672, Gibco; Thermo Fisher Scientific, Inc.) in the colon tissues of the mice, according to the manufacturer's instructions. The OD value was measured at $450 \mathrm{~nm}$ using a microplate reader (Thermo Fisher Scientific, Inc.) and the concentration was calculated using a standard curve.

Hematoxylin and eosin $(H \& E)$ staining. Colonic mucosal NKT cell infiltration was observed using H\&E staining. The Chiu's scoring system was used to quantitatively determine the histological scores of the intestine (20). Following 7 days of growth, the mice were sacrificed by decapitation and $10 \mathrm{~cm}$ colonic mucosa was removed from four mice in each group, washed with PBS and fixed in 10\% natural buffered formalin at room temperature for $30 \mathrm{~min}$. The fixed tissues were embedded in paraffin, cut into tissue sections (5- $\mu \mathrm{m}$ thick) and stained with $\mathrm{H} \& \mathrm{E}\left(25^{\circ} \mathrm{C} ; 3 \mathrm{~h}\right.$; Beyotime Institute of Biotechnology). The slides were examined with a light microscope (Nikon Corporation) and the data derived were qualitatively evaluated with QWin software 3.1 (Leica Microsystems, Inc.) using the following parameter: NKT cell infiltration.

Western blot analysis. Frozen colonic tissues in liquid nitrogen were mechanically homogenized $(20 \% \mathrm{w} / \mathrm{v})$ in lysis buffer ( $\mathrm{pH}$ 7.4) containing $0.1 \mathrm{mM}$ EDTA, $20 \mathrm{mM}$ HEPES, $12.5 \mathrm{mM} \mathrm{MgCl}, 150 \mathrm{mM} 0.1 \%$ Nonidet P40, $\mathrm{NaCl}, 1 \mathrm{mM}$ dithiothreitol, $0.2 \mathrm{mM}$ phenylmethylsulfonyl fluoride, and $1 \mu \mathrm{g} / \mathrm{ml}$ leupeptin, aprotinin and pepstatin. Homogenates were sonicated $\left(120 \mathrm{~Hz} ; 4^{\circ} \mathrm{C} ; 20 \mathrm{~min}\right)$ and subsequently centrifuged for $10 \mathrm{~min}$ at $12,000 \mathrm{xg}$ at $4^{\circ} \mathrm{C}$. Protein concentration was assessed using a BCA assay kit (Beyotime Institute of Biotechnology). Equivalent quantities $(20 \mu \mathrm{g})$ of protein from the cell lysates were loaded onto 15\% gels (Beyotime Institute of Biotechnology), separated via SDS-PAGE and then separated proteins were transferred onto polyvinylidene fluoride membranes (EMD Millipore). The membranes were blocked with $5 \%$ non-fat milk in Tris-buffered saline $\left(25^{\circ} \mathrm{C}\right.$; $30 \mathrm{~min})$ at room temperature and subsequently incubated with primary antibodies $\left(4^{\circ} \mathrm{C}\right.$; overnight $)$ against Beclin-1 (1:1,000; cat. no. 3738; Cell Signaling Technology, Inc.), LC3B (1:1,000; cat. no. 43566; Cell Signaling Technology, Inc.), PI3K (1:1,000; cat. no. 4255; Cell Signaling Technology, Inc.), Akt (1:1,000; cat. no. 4691; Cell Signaling Technology, Inc.), mTORC1 (1:1,000; cat. no. 10441-1-AP; ProteinTech Group, Inc.), phosphorylated (p)-MTORC1 (1:1,000; cat. no. 5536; 
Cell Signaling Technology, Inc.), ATG13 (1:1,000; cat. no. 13273; Cell Signaling Technology, Inc.), Occludin (1:1,000; cat. no. 91131; Cell Signaling Technology, Inc.), ZO-1 (1:1,000; cat. no. ab59720; Abcam), claudin-2 (1:1,000; cat. no. 48120; Cell Signaling Technology, Inc.) and GAPDH (1:1,000; cat. no. 5174; Cell Signaling Technology, Inc.) overnight at $4^{\circ} \mathrm{C}$. The following day, the membranes were rinsed using TBS with $1 \%$ Tween-20 three times for 8 min each, followed by incubation at room temperature for $1 \mathrm{~h}$ with goat anti-rabbit horseradish peroxidase-conjugated secondary antibody (1:1,000; Cell Signaling Technology, \#7074, Inc.). The membranes were washed three times and detected using ECL reagent (1:1,000; Cell Signaling Technology, Inc.). Finally, the densitometry was analyzed by Image J v1.8.0.112 software (National Institutes of Health).

Immunofluorescence. $\mathrm{LC} 3 \mathrm{~B}$ expression levels were analyzed by immunofluorescence in colonic tissues. The $2-\mu \mathrm{m}$ paraffin-embedded sections of the colon tissue sections $(4 \%$ PFA; $25^{\circ} \mathrm{C} ; 24 \mathrm{~h}$ ) were dewaxed, rehydrated with gradient alcohol, subjected to antigen repair $\left(100^{\circ} \mathrm{C}\right)$ and subsequently rinsed three times with $0.01 \mathrm{M}$ PBS $\left(25^{\circ} \mathrm{C}\right)$ with $0.1 \%$ Tween-20 (PBST). The sections were blocked $\left(25^{\circ} \mathrm{C}\right)$ with $5 \% \mathrm{BSA}$ and $0.3 \%$ Triton X-100 for $1 \mathrm{~h}$, placed in a wet box for an additional $1 \mathrm{~h}$ and incubated with a primary antibody against LC3B (1:1,000; cat. no. 43566; Cell Signaling Technology, Inc.) at $4{ }^{\circ} \mathrm{C}$ overnight. The following day, the samples were washed three times with PBS for 5 min each time and then was incubated with Alexa-488-conjugated donkey secondary antibody against rabbit antibody (1:3,000; Cell Signaling Technology, Inc.; cat. no. 4412). The nuclei were counterstained with $10 \mu \mathrm{g} / \mathrm{ml}$ DAPI $\left(25^{\circ} \mathrm{C} ; 3 \mathrm{~min}\right)$ at room temperature. The images were captured using an Olympus fluorescent microscope (Olympus Corporation).

Statistical analysis. The results are presented as the mean \pm standard deviation. All data presented are representative of $\geq 3$ experiments. Statistical analysis was conducted using GraphPad Prism 6.2 software (GraphPad Software, Inc.) and significance was determined using two-way ANOVA and Dunnett's multiple comparisons test. $\mathrm{P}<0.05$ was considered to indicate a statistically significant difference.

\section{Results}

$P D$ alleviates the symptoms of UC in mice. In order to investigate the therapeutic effect of PD on colitis, the present study induced colitis in a mouse model by skin pre-sensitization and colonic administration of oxazolone. The results indicated that the mice injected with oxazolone developed diarrhea, bloody stool and weight loss. Histological changes, such as monocyte infiltration, intestinal wall thickening, mucosal hyperplasia and distortion of crypt structures, were observed in the model group compared with the normal control group (Fig. 1A). Concomitantly, it was found that PD significantly reduced H\&E and DAI scores compared with the control (Tables I and II). These effects were also noted with IL-13 intervention and oral administration of 5-ASA (Fig. 1A-C). These findings indicated that PD mitigated the severity of oxazolone-induced colitis in mice.
Table I. Damage to the intestinal mucosa was graded using Chiu's scoring system.

\begin{tabular}{lll}
\hline Feature & Score & \multicolumn{1}{c}{ Description } \\
\hline Inflammation severity & 0 & None \\
& 1 & Mild \\
& 2 & Moderate \\
Inflammation extent & 3 & Severe \\
& 0 & None \\
& 1 & Mucosa \\
Crypt damage & 2 & Submucosa \\
& 3 & Transmural \\
& 0 & None \\
& 1 & Basal 1/3 damage \\
& 2 & Basal 2/3 damage \\
& 3 & Crypt lost; surface \\
& & epithelium present \\
& 4 & Crypt and surface \\
& & epithelium lost \\
& & $0 \%$ \\
& & $1-25 \%$ \\
& & $26-50 \%$ \\
& & $51-75 \%$ \\
& & $76-100 \%$ \\
& &
\end{tabular}

Table II. DAI scoring system in oxazolone-induced colitis mice.

\begin{tabular}{lcll}
\hline Score & $\begin{array}{c}\text { Weight } \\
\text { loss, } \%\end{array}$ & $\begin{array}{c}\text { FOB/bloody } \\
\text { stool }\end{array}$ & $\begin{array}{c}\text { Stool } \\
\text { consistency }\end{array}$ \\
\hline 0 & None & Normal & Normal \\
1 & $1-5$ & Loose stools & FOB (+) \\
2 & $5-10$ & Diarrhea & $\begin{array}{l}\text { Bloody } \\
\text { stool }\end{array}$ \\
3 & $10-20$ & & \\
\hline
\end{tabular}

DAI, disease activity index; FOB, fecal occult blood.

PD inhibits oxazolone-induced colitis inflammation. The length of the colon is considered an indirect indicator of inflammation and is negatively correlated with the severity of colitis (21). In addition, the effects of PD were investigated on inflammation of oxazolone-induced colitis. The colon tissues were significantly longer and lighter in the normal control, IL-13 intervention, 5-ASA and PD groups compared with those of the model group; Fig. 2A-C). The accumulation of MPO in the colon is a marker of neutrophil entry into the tissue and IL-13 is an inflammatory cytokine characteristic of UC. The ELISA results indicated that PD caused a significant reduction in the levels of MPO in the colon tissues and a significant decrease in serum IL-13 levels compared with the model group (Fig. 3A and B). The same results were found 
A

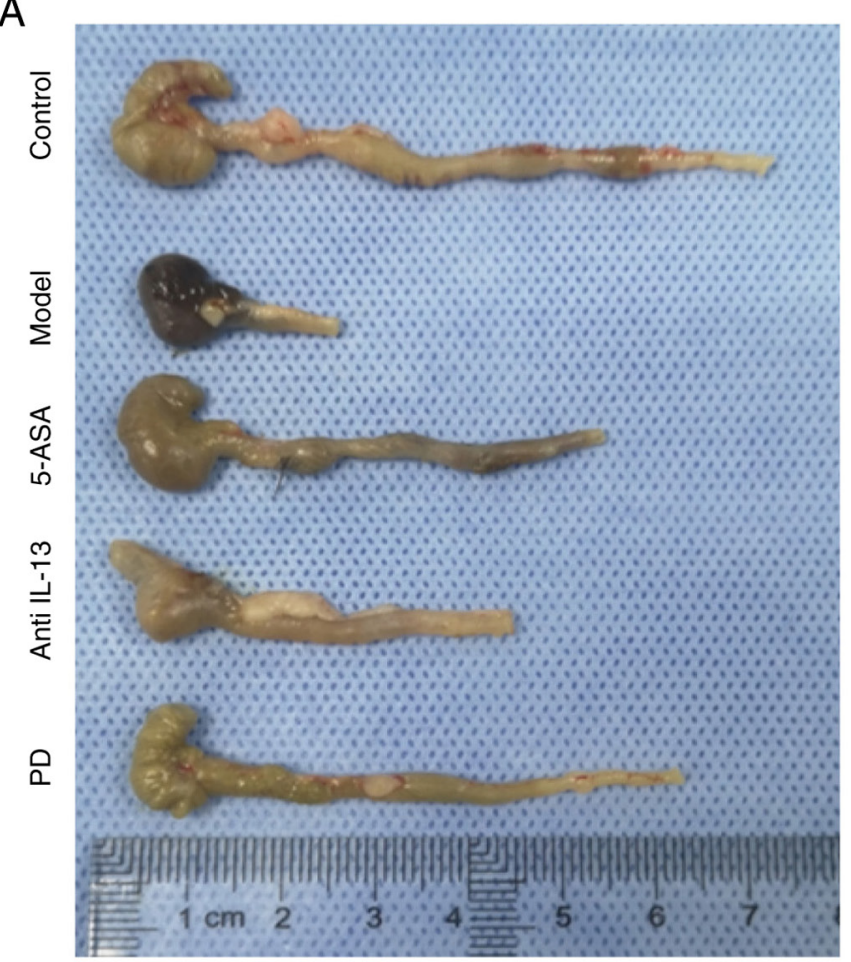

B
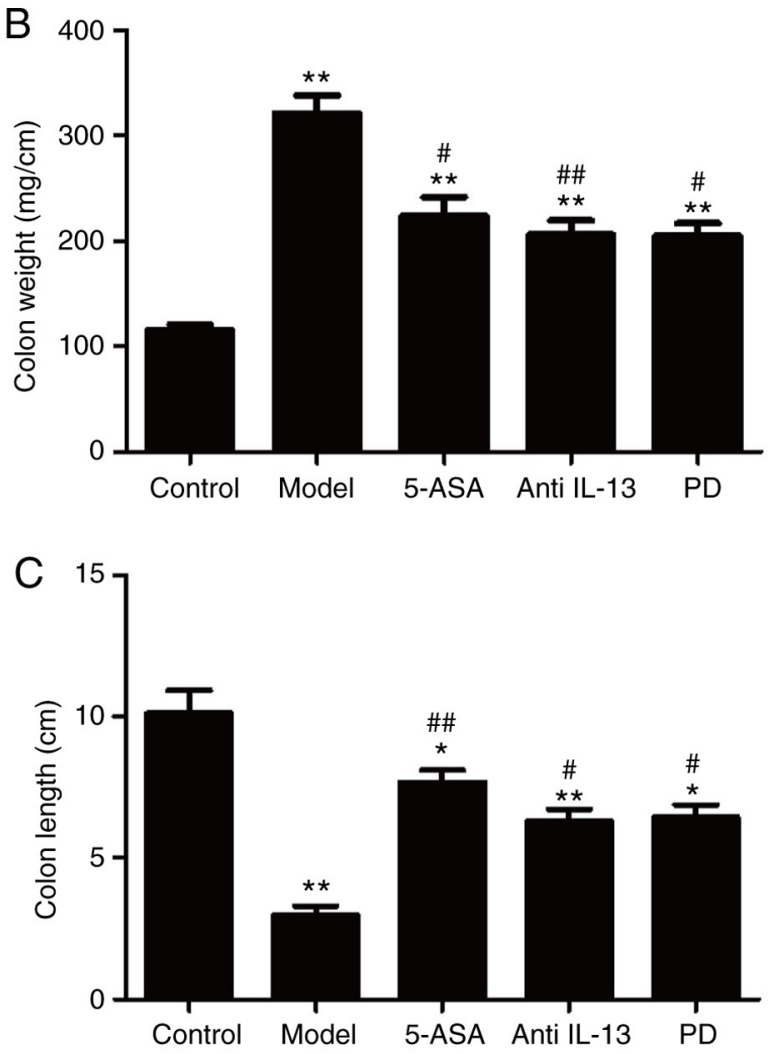

Figure 2. PD reduces colon weight and length. (A) The length and weight were evaluated from the different groups of mice. Statistical analysis of colon (B) weight and (C) length. The data are representative of three independent experiments and are expressed as the mean \pm standard deviation. ${ }^{*} \mathrm{P}<0.05$, ${ }^{* *} \mathrm{P}<0.01$ vs. normal control group; ${ }^{\#} \mathrm{P}<0.05,{ }^{\# \#} \mathrm{P}<0.01$ vs. model control group. $\mathrm{PD}$, Pulsatilla decoction; 5-ASA, 5-aminosalicylic acid.
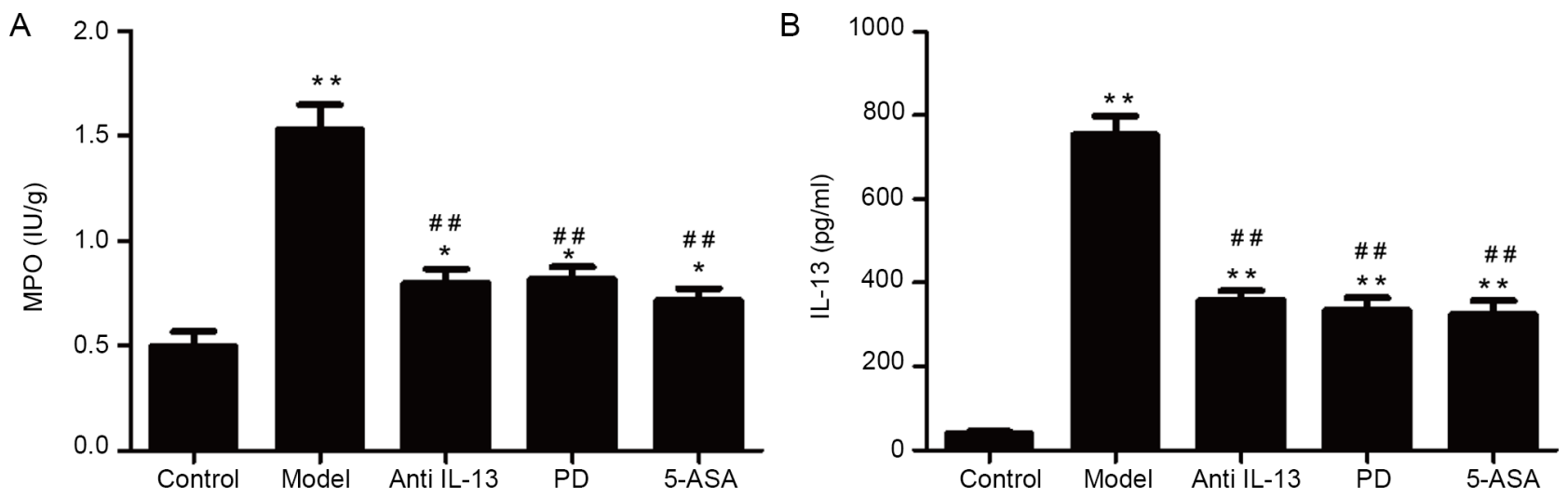

Figure 3. PD inhibits ulcerative colitis inflammatory response. Expression levels of the inflammatory cytokines (A) MPO and (B) IL-13 were determined in colon tissues by ELISA. The data are representative of three independent experiments and are expressed as the mean \pm standard deviation. ${ }^{*} \mathrm{P}<0.05$, ${ }^{* *} \mathrm{P}<0.01$ vs. normal control group; ${ }^{\# \#} \mathrm{P}<0.01$ vs. model control group. PD, Pulsatilla decoction; MPO, myeloperoxidase; 5-ASA, 5-aminosalicylic acid.

in the IL-13 and 5-ASA groups compared with model group (Fig. 3A and B). These findings illustrated that PD could inhibit oxazolone-induced colitis inflammation.

$P D$ inhibits the activation of autophagy by regulating $L C 3$ and Beclinl expression. To assess the levels of autophagy in the colon tissues, western blotting, immunofluorescence and RT-qPCR assays confirmed that the percentage of cells with low LC3 fluorescence intensity was significantly higher compared with that of the normal control group (Fig. 4A and B). The mRNA and protein expression levels of Beclin1 and LC3 were upregulated in the model group compared with control (Fig. 5A-E). However, Beclin1 and LC3 expression levels significantly decreased when the mice were administered PD, 5-ASA or IL-13 (Fig. 5A-E). This indicated that PD significantly inhibited tissue autophagy in oxazolone-induced colitis. In order to further investigate the molecular mechanism of action of PD, the expression levels of the associated molecules in the PI3K-Akt-mTORC1 signaling pathway were investigated via western blotting and RT-qPCR assays. The results demonstrated that PI3K, Akt, mTORC1 and ATG13 expression levels were significantly downregulated in the PD, 5-ASA or IL-13 intervention groups (Fig. 6A-D). In 

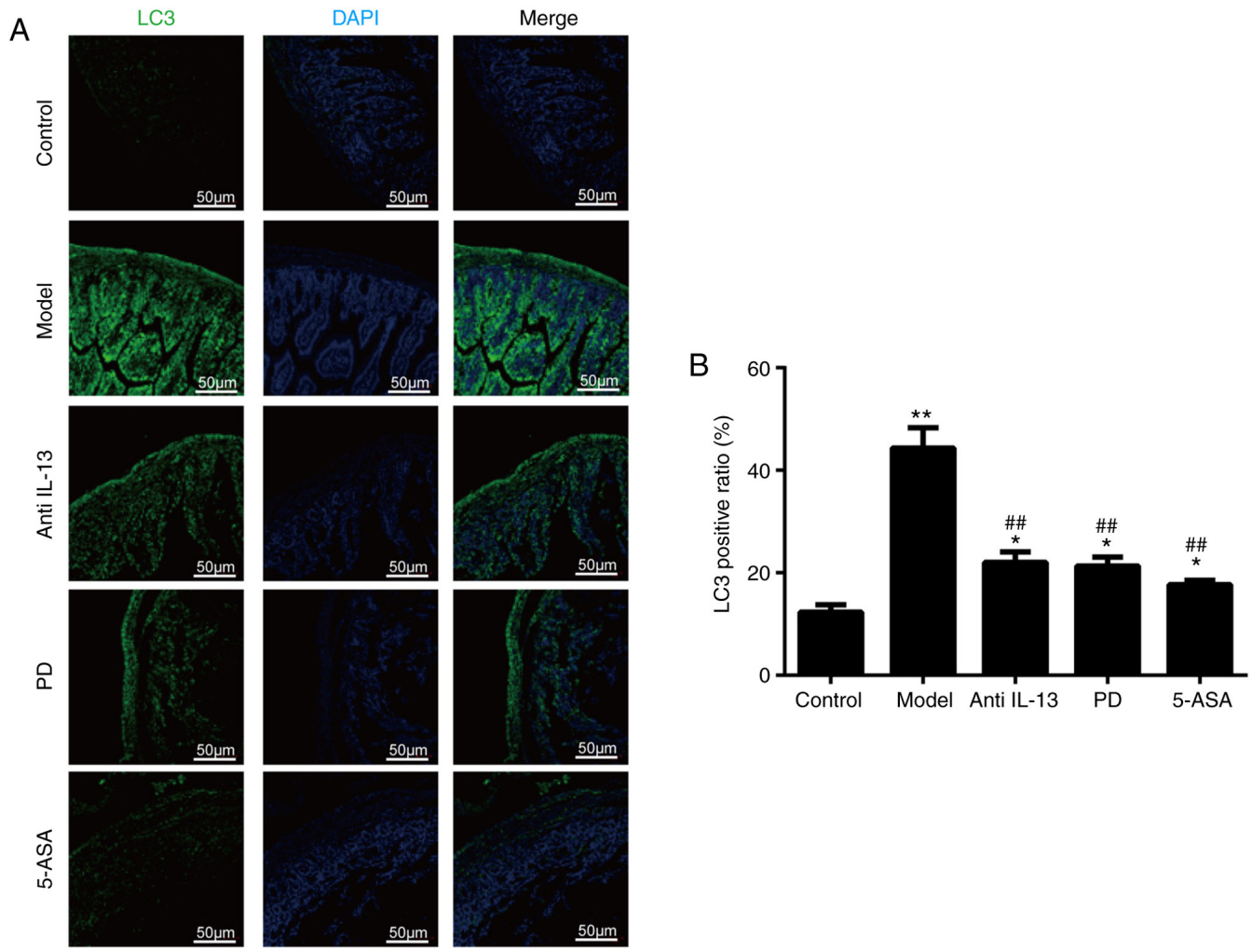

Figure 4. Effect of PD on autophagy in oxazolone-induced colitis tissue. (A and B) Expression levels of LC3 in the colon tissues of oxazolone-induced colitis mice treated with different drugs were detected using immunofluorescence analysis. The data are representative of three independent experiments and are expressed as the mean \pm standard deviation. ${ }^{*} \mathrm{P}<0.05,{ }^{* * *} \mathrm{P}<0.01$ vs. normal control group; ${ }^{\# \#} \mathrm{P}<0.01$ vs. model control group. $\mathrm{PD}$, $\mathrm{Pulsatilla} \mathrm{decoction;}$ 5-ASA, 5-aminosalicylic acid.

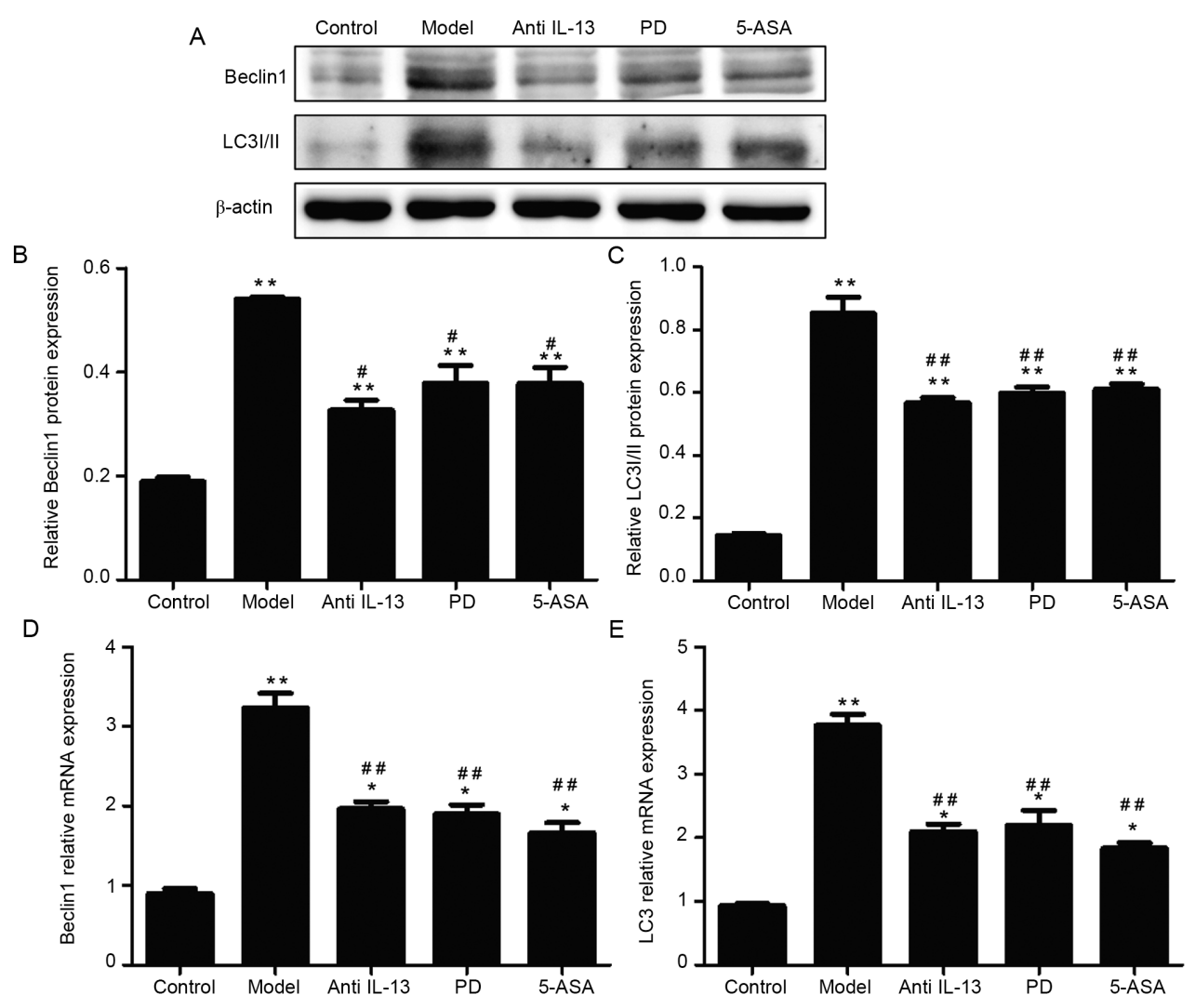

Figure 5. PD downregulates the expression levels of autophagy-associated genes and proteins. Detection of autophagy-associated expression via (A-C) western blotting and (D and E) reverse transcription-quantitative PCR. The data are representative of three independent experiments and are expressed as the mean \pm standard deviation. ${ }^{~} \mathrm{P}<0.05^{* *} \mathrm{P}<0.01$ vs. normal control group; ${ }^{*} \mathrm{P}<0.05,{ }^{\# \#} \mathrm{P}<0.01$ vs. model control group. $\mathrm{PD}$, $\mathrm{Pulsatilla}$ decoction; 5-ASA, 5-aminosalicylic acid. 

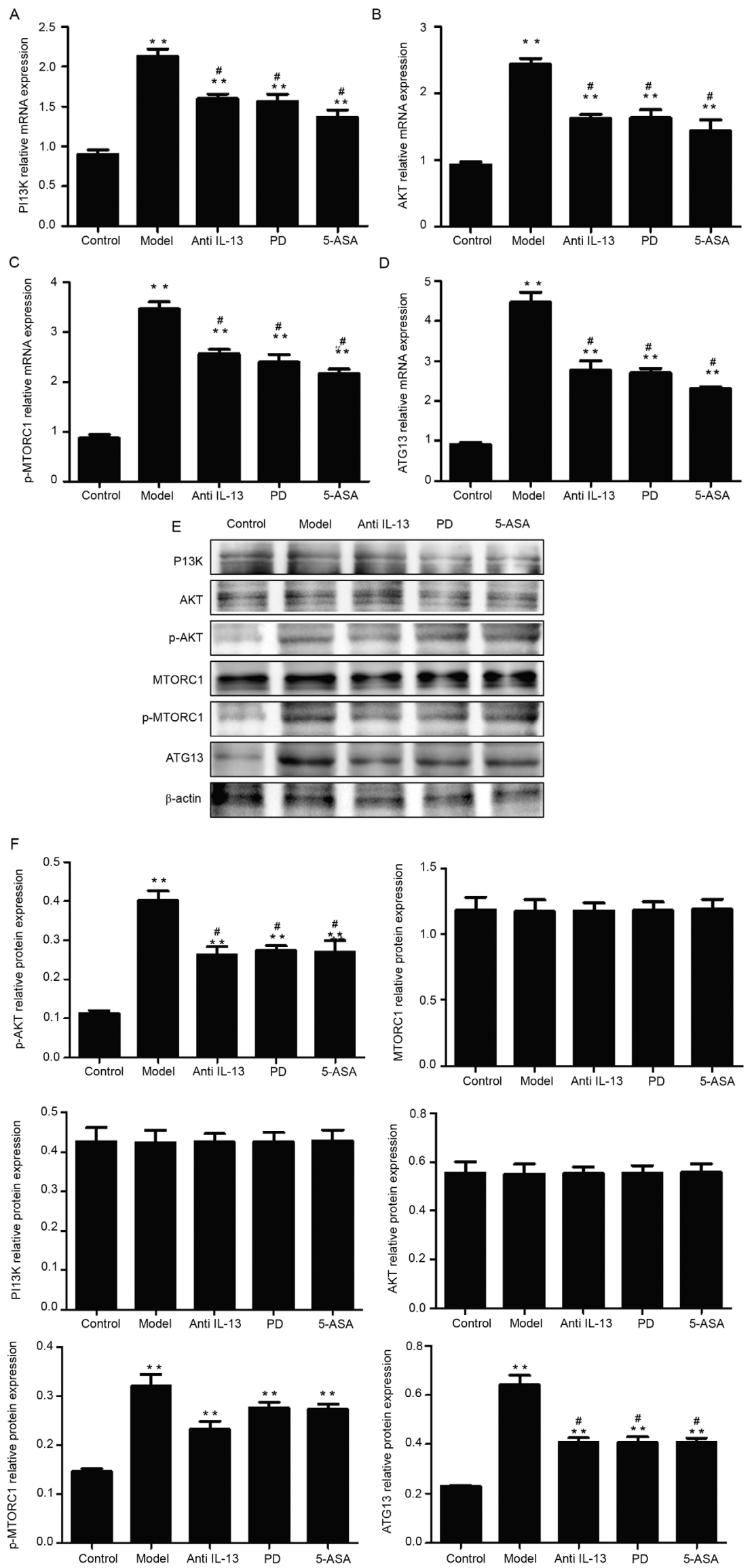

Figure 6. Protective effects of PD against oxazolone-induced colitis are exerted via regulation of the PI3K-Akt-mTORC1 signaling pathway. (A-D) Assessment of the mRNA expression levels of the PI3K-Akt-mTORC1 signaling pathway members. (E and F) Protein expression of the PI3K-Akt-mTORC1 signaling pathway was detected by western blot analysis. The data are representative of three independent experiments and are expressed as the mean \pm standard deviation. ${ }^{* *} \mathrm{P}<0.01$ vs. normal control group; ${ }^{\#} \mathrm{P}<0.05$ vs. model control group. $\mathrm{PD}$, Pulsatilla decoction; 5-ASA, 5-aminosalicylic acid; p-, phosphorylated; ATG13, autophagy-related protein 13 . 

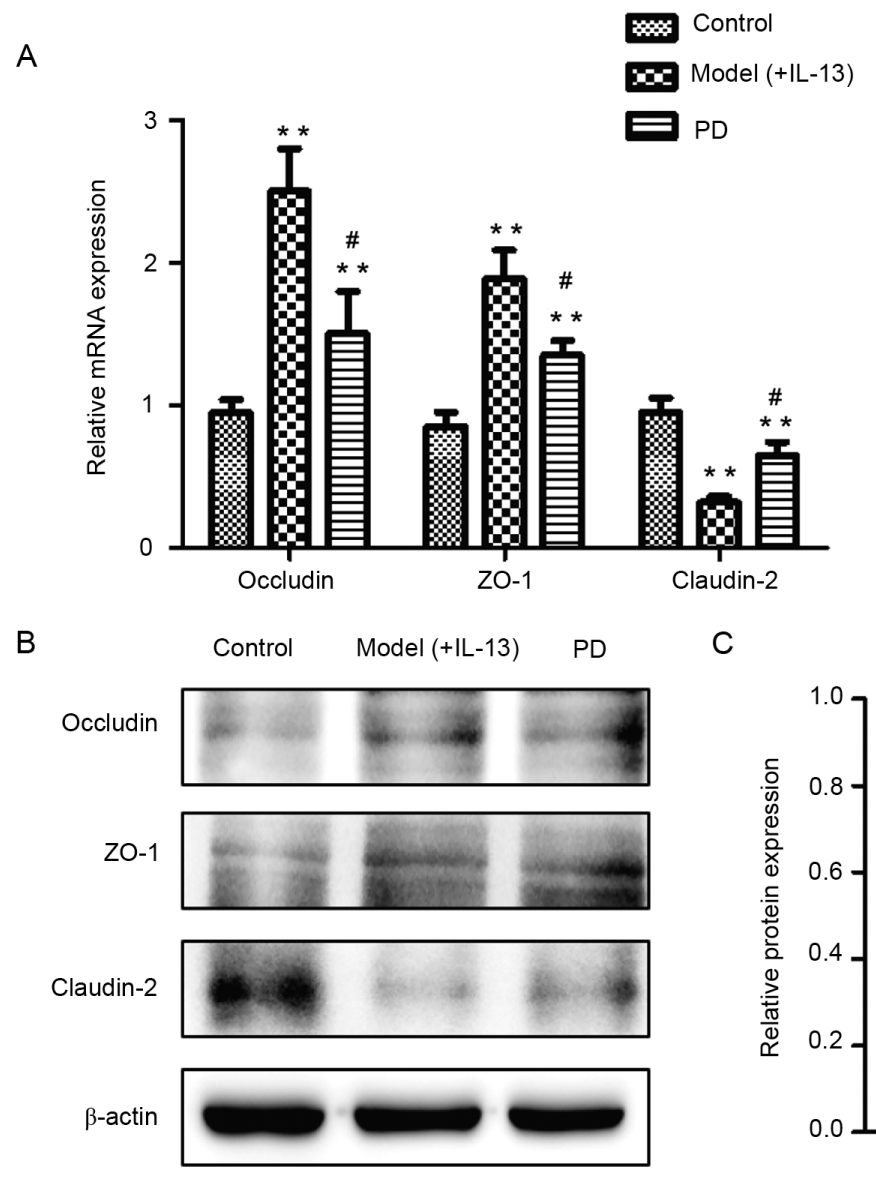

C

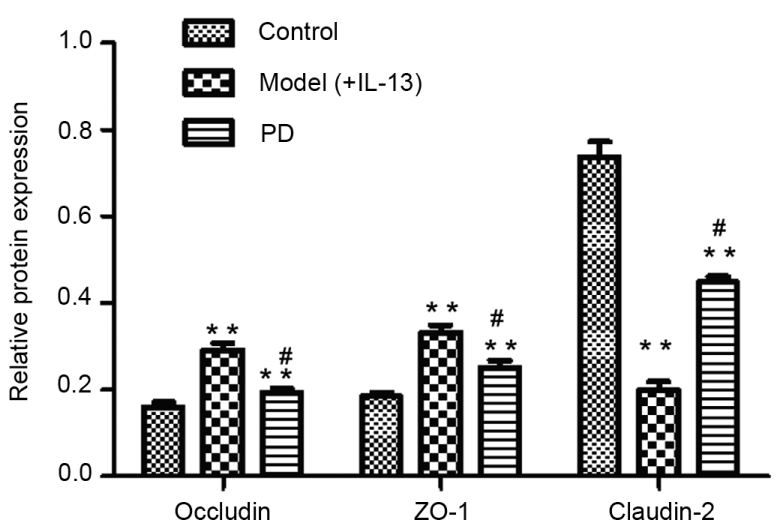

Figure 7. PD protects the colon epithelial barrier through alteration of tight junction proteins. (A) Reverse transcription-quantitative PCR analysis of occludin, ZO-1 and claudin-2 in oxazolone-induced colitis tissues. (B and C) Occludin, ZO-1 and claudin-2 expression levels were detected by western blot analysis. The data are representative of three independent experiments and are expressed as the mean \pm standard deviation. ${ }^{* *} \mathrm{P}<0.01$ vs. normal control group; ${ }^{*} \mathrm{P}<0.05 \mathrm{vs}$. model (anti IL-13) group. PD, Pulsatilla decoction; ZO-1, zona occludens protein 1.

addition, western blot analysis indicated that the of p-AKT, p-mTORC1 and ATG13 expression levels were increased in the model group compared with the control group; however, expression levels were decreased in the PD, 5-ASA and IL-13 groups compared with the model group. There was no change in the expression levels of Akt or mTORC1.

(Fig. 6E and F). Therefore, these results suggested that the protective effects of PD against oxazolone-induced colitis were exerted via the regulation of the PI3K-Akt-mTORC1 signaling pathway.

$P D$ protects the colon epithelial barrier through alteration of tight junction protein expression. Several studies have shown that the expression levels of tight junction proteins found between intestinal epithelial cells, are affected in inflammatory bowel disease, leading to alterations in tight junction structure and function $(10,22)$. Therefore, to determine whether PD maintains the integrity of the intestinal barrier, occludin and ZO-1 expression levels were detected. The data indicated that they were decreased, whereas claudin-2 levels were increased compared with model (Fig. 7A-C). The relative mRNA expression levels of these markers including occludin and ZO-1were altered compared with model (Fig. 7A) following PD treatment. These results suggested that PD protected the intestinal barrier by altering the expression levels of the colonic tissue tight junction proteins, which were induced by inflammatory stimuli.

\section{Discussion}

Numerous clinical studies have shown that TCM has unique advantages in the treatment of UC and that the majority of the herbal preparations exhibit optimal clinical therapeutic significance $(8,23)$. The treatment process of UC involves immune regulation and modulation of cytokine levels, signaling pathways and associated gene expression levels (18). These molecular events are interconnected through a variety of mechanisms in UC, so as to relieve intestinal inflammation, restore the normal structure and function of the colonic mucosa and achieve optimal treatment efficacy (24-27). The use of PD was first recorded $\sim 1,800$ years ago by Zhang Zhongjing in his book 'Treatise on Febrile Diseases', and was a famous prescription for treating eczema $(16,28)$. Therefore, in the present study, the efficacy of PD was briefly assessed in oxazolone-induced colitis and its molecular mechanisms were examined with regard to the modulation of the PI3K-Akt-mTORC1 and autophagy-related signaling pathways.

In the present study, the serum levels of IL-13 and the colon tissue levels of MPO were measured in mice with UC 
in order to investigate the possible immunomodulatory effects of PD on UC. The results indicated that IL-13 and MPO levels in model mice were significantly higher than those in the control group, suggesting that increased IL-13 and MPO levels were consistent with intestinal mucosal injury and inflammation and that they could serve as an indicator of UC activity, which was consistent with the results reported in previous studies (29-32). These data also verified that proinflammatory cytokines caused the intestinal mucosa to produce an excessive inflammatory response, thereby triggering intestinal injury. The expression levels of IL-13 and MPO were inhibited and the histological and DAI scores were reduced in the treatment group. The weight and length of the colon tissue were significantly decreased in the treatment group, indicating that this effect was consistent with previous findings (16). Overall, the results highlighted that PD could effectively alleviate the inflammation and severity of colitis in mice.

Numerous studies that examined the efficacy of herbal products were not successful in evaluating their mechanisms of action (14), which hindered their widespread application. Based on the results of the present study showing reduced inflammation following PD treatment, it was speculated that the therapeutic effect of PD may be associated with the modulation of the intracellular signaling transduction pathway. It has been shown that the PI3K-Akt-mTORC1 signaling pathway is also involved in the differentiation and maturation of iNKT cells (33-36). To assess whether the PI3K-Akt-mTORC1 signaling pathway is involved in regulating inflammation, the mRNA and protein expression levels of LC3, Beclin1 and several of the PI3K-Akt-mTORC1 signaling pathway members were assessed via RT-qPCR, western blotting and immunofluorescence analyses. Following PD or IL-13 administration, both mRNA and protein phosphorylation levels of the PI3K-Akt-mTORC1 signaling pathway were significantly decreased, and total protein expression was not influenced. The results revealed that the expression levels of these target genes were significantly increased in the model group compared with those noted in the control group, indicating that the PI3K-Akt-mTORC1 signaling pathway was activated in the UC model group.

Previous studies have reported that impaired expression of intestinal epithelial tight junction proteins in inflammatory bowel disease leads to alterations in tight junction structure and function, resulting in damage to the intestinal epithelial barrier (37-39). Subsequently, it has been demonstrated that PD can repair the damaged intestinal epithelial barrier by regulating the expression levels of junction proteins (20). In the present study, these findings were verified by PD or IL-13 neutralization, which downregulated occludin and ZO-1 protein expression, and upregulated the expression levels of claudin-2, as confirmed via RT-qPCR and western blotting.

In summary, the present study elucidated the in vivo therapeutic effect of PD on UC model mice by a potential mechanism that may be associated with the activation of the PI3K-Akt-mTORC1 signaling pathway. The data lay the experimental foundation for PD as a promising treatment option for UC, however whether the mechanism of action of PD is related to autophagy, requires further exploration in the future. The research and development of UC herbal preparations must follow the developmental trends of TCM and should aim to accelerate technological innovation and transformation to develop modern Chinese medicine preparations that meet market needs and possess successful therapeutic effects.

\section{Acknowledgements}

Not applicable.

\section{Funding}

The present study was supported by Putuo District of Shanghai Science and Technology Commission Research Project (grant no. ptkwws201806) and Project of Shanghai Municipal Health Commission of China (grant no. 202040159).

\section{Availability of data and materials}

The datasets used and/or analyzed during the current study are available from the corresponding author on reasonable request.

\section{Authors' contributions}

QC and XW participated in the study design, manuscript writing and confirm the authenticity of all the raw data. LX performed experiments, analyzed data and participated in manuscript writing. TW and JX participated in statistical analysis and manuscript writing. FF, YZ and JW performed experiments and analyzed data. All authors read and approved the final manuscript.

\section{Ethics approval and consent to participate}

Ethical approval was given by the Ethics Committee of Shanghai University of Traditional Chinese Medicine (approval no. 0015; Shanghai, China).

\section{Patient consent for publication}

Not applicable.

\section{Competing interests}

The authors declare that they have no competing interests.

\section{References}

1. Feuerstein JD, Moss AC and Farraye FA: Ulcerative Colitis. Mayo Clin Proc 94: 1357-1373, 2019.

2. Rubin DT, Ananthakrishnan AN, Siegel CA, Sauer BG and Long MD: ACG Clinical Guideline: Ulcerative Colitis in Adults. Am J Gastroenterol 114: 384-413, 2019.

3. Nakov R: New markers in ulcerative colitis. Clin Chim Acta 497: 141-146, 2019.

4. Ko CW, Singh S, Feuerstein JD, Falck-Ytter C, Falck-Ytter Y and Cross RK; American Gastroenterological Association Institute Clinical Guidelines Committee: AGA Clinical practice guidelines on the management of Mild-to-Moderate ulcerative colitis. Gastroenterology 156: 748-764, 2019.

5. Cross R, Ko CW and Singh S: Mild-to-Moderate ulcerative colitis guideline. Gastroenterology 156: 768, 2019.

6. Teschke R, Wolff A, Frenzel C, Eickhoff A and Schulze J: Herbal traditional Chinese medicine and its evidence base in gastrointestinal disorders. World J Gastroenterol 21: 4466-4490, 2015. 
7. Fan Y, Yi W, Huang H, Mei Z and Feng Z: Efficacy of herbal medicine (Gegen Qinlian Decoction) on ulcerative colitis: A systematic review of randomized controlled trials. Medicine (Baltimore) 98: e18512, 2019.

8. Shen Z, Zhou Q, Ni Y, He W, Shen H and Zhu L: Traditional Chinese medicine for mild-to-moderate ulcerative colitis: Protocol for a network meta-analysis of randomized controlled trials. Medicine 98: e16881, 2019.

9. Lin JC, Wu JQ, Wang F, Tang FY, Sun J, Xu B, Jiang M, Chu Y Chen D, Li X, et al: QingBai decoction regulates intestinal permeability of dextran sulphate sodium-induced colitis through the modulation of notch and NF- $\kappa$ B signalling. Cell Prolif 52: e12547, 2019.

10. Wang XM, Tian G, Duan QJ, Wu DQ, Shao J, Wang TM and Wang CZ: Therapeutic potential of n-butanol extract of Pulsatilla decoction in a murine model of ulcerative colitis induced by DSS combined with Candida albicans colonization. Zhongguo Zhong Yao Za Zhi 43: 2979-2984, 2018 (In Chinese).

11. Wang WH, Zhan ZL, Peng HS, Yang J and Qian JP: Textual research on the origin and producing area of Baitouweng (Radix Pulsatillae). Zhonghua Yi Shi Za Zhi 47: 14-18, 2017 (In Chinese).

12. Yu Z, Liu HJ, Dun HH, Dong Q and Liang C: Effect of Pulsatilla decoction on the expression of proinflammatory cytokines in inflammatory bowel disease. Zhongguo Ying Yong Sheng Li Xue Za Zhi 27: 416-419, 2011 (In Chinese).

13. Pontoglio M: Hepatocyte nuclear factor 1, a transcription factor at the crossroads of glucose homeostasis. J Am Soc Nephrol 11 (Suppl 16): S140-S143, 2000

14. Wang X, Fan F and Cao Q: Modified Pulsatilla decoction attenuates oxazolone-induced colitis in mice through suppression of inflammation and epithelial barrier disruption. Mol Med Rep 14 1173-1179, 2016.

15. Lu SW, Liu HJ, Zhao W, Li L, Dun HH and Liang C: Molecular mechanisms involved in the treatment of inflammatory bowel disease by Pulsatilla decoction. Zhongguo Ying Yong Sheng Li Xue Za Zhi 27: 106-109, 2011 (In Chinese).

16. Hua YL, Ma Q, Zhang XS, Jia YQ, Peng XT, Yao WL, Ji P, Hu JJ and Wei YM: Pulsatilla decoction can treat the dampness-heat diarrhea rat model by regulating glycerinphospholipid metabolism based lipidomics approach. Front Pharmacol 11: 197, 2020.

17. Liu X, He S, Li Q, Mu X, Hu G and Dong H: Comparison of the Gut microbiota between pulsatilla decoction and levofloxacin hydrochloride therapy on Escherichia coli Infection. Front Cell Infect Microbiol 10: 319, 2020

18. Wang X, Yang J, Cao Q and Tang J: Therapeutic efficacy and mechanism of water-soluble extracts of Banxiaxiexin decoction on BALB/c mice with oxazolone-induced colitis. Exp Ther Med 8: 1201-1204, 2014.

19. Livak KJ and Schmittgen TD: Analysis of relative gene expression data using real-time quantitative PCR and the 2(-Delta Delta C(T)) method. Methods 25: 402-408, 2001.

20. Ji AL, Li T, Zu G, Feng DC, Li Y, Wang GZ, Yao JH and Tian XF: Ubiquitin-specific protease 22 enhances intestinal cell proliferation and tissue regeneration after intestinal ischemia reperfusion injury. World J Gastroenterol 25: 824-836, 2019.

21. Gordon IO, Agrawal N, Willis E, Goldblum JR, Lopez R, Allende D, Liu X, Patil DY, Yerian L, El-Khider F, et al: Fibrosis in ulcerative colitis is directly linked to severity and chronicity of mucosal inflammation. Aliment Pharmacol Ther 47: 922-939, 2018.

22. Qian XX, Cai CW, Li HY, Lai LJ, Song DJ, Qiao YQ, Shen J and Ran ZH: Transcribed ultraconserved region (T-UCR) uc.261 expression is closely correlated with disease activity and intestinal permeability in Crohn's disease. Ther Adv Gastroenter 12: 1756284819880733,2019

23. Guo BJ, Bian ZX, Qiu HC, Wang YT and Wang Y: Biological and clinical implications of herbal medicine and natural products for the treatment of inflammatory bowel disease. Ann N Y Acad Sci 1401: 37-48, 2017
24. Suh SY and An WG: Systems pharmacological approach of pulsatillae radix on treating Crohn's disease. Evid Based Complement Alternat Med 2017: 4198035, 2017.

25. Wang Q, Su CP, Zhang HM, Ren YL, Wang W and Guo SZ: Anti-inflammatory mechanism of heat-clearing and detoxifying Chinese herbs. Zhongguo Zhong Yao Za Zhi 43: 3787-3794, 2018 (In Chinese).

26. Li S, Li X, Yang R, Wang B, Li J, Cao L, Xiao S and Huang W: Effects of anemoside B4 on pharmacokinetics of florfenicol and mRNA expression of CXR, MDR1, CYP3A37 and UGT1E in broilers. J Vet Med Sci 81: 1804-1809, 2019.

27. Shi Y, Zhao M, Yao H, Yang P, Xin T, Li B, Sun W and Chen S: Rapidly discriminate commercial medicinal Pulsatilla chinensis (Bge.) Regel from its adulterants using ITS2 barcoding and specific PCR-RFLP assay. Sci Rep 7: 40000, 2017.

28. Frisardi V, Panza F, Seripa D, Farooqui T and Farooqui AA Glycerophospholipids and glycerophospholipid-derived lipid mediators: A complex meshwork in Alzheimer's disease pathology. Prog Lipid Res 50: 313-330, 2011.

29. Tatiya-Aphiradee N, Chatuphonprasert W and Jarukamjorn K Immune response and inflammatory pathway of ulcerative colitis. J Basic Clin Physiol Pharmacol 30: 1-10, 2018.

30. Salem HA and Wadie W: Effect of Niacin on Inflammation and Angiogenesis in a Murine Model of Ulcerative Colitis. Sci Rep 7: 7139, 2017.

31. Leung VSY, Benoit-Biancamano MO and Pang DSJ: Performance of behavioral assays: The Rat Grimace Scale, burrowing activity and a composite behavior score to identify visceral pain in an acute and chronic colitis model. Pain Rep 4 e718, 2019.

32. Hanauer SB: Oral or topical 5-ASA in ulcerative colitis. Dig Dis 34: 122-124, 2016.

33. Lai LJ, Shen J and Ran ZH: Natural killer T cells and ulcerative colitis. Cell Immunol 335: 1-5, 2019.

34. Chang HK and Hou WS: Retinoic acid modulates interferon- $\gamma$ production by hepatic natural killer $\mathrm{T}$ cells via phosphatase $2 \mathrm{~A}$ and the extracellular signal-regulated kinase pathway. J Interferon Cytokine Res 35: 200-212, 2015.

35. Wu S, Yang C, Xu N, Wang L, Liu Y, Wang J and Shen X: The protective effects of Helix B surface peptide on experimental acute liver injury induced by carbon tetrachloride. Dig Dis Sci 62: 1537-1549, 2017.

36. Zhang L, Tschumi BO, Corgnac S, Rüegg MA, Hall MN, Mach JP, Romero P and Donda A: Mammalian target of rapamycin complex 1 orchestrates invariant NKT cell differentiation and effector function. J Immunol 193: 1759-1765, 2014.

37. Landy J, Ronde E, English N, Clark SK, Hart AL, Knight SC, Ciclitira PJ and Al-Hassi HO: Tight junctions in inflammatory bowel diseases and inflammatory bowel disease associated colorectal cancer. World J Gastroenterol 22: 3117-3126, 2016.

38. Chen T, Xue H, Lin R and Huang Z: MiR-34c and PlncRNA1 mediated the function of intestinal epithelial barrier by regulating tight junction proteins in inflammatory bowel disease. Biochem Biophys Res Commun 486: 6-13, 2017.

39. He C, Deng J, Hu X, Zhou S, Wu J, Xiao D, Darko KO, Huang Y, Tao T, Peng M, et al: Vitamin A inhibits the action of LPS on the intestinal epithelial barrier function and tight junction proteins. Food Funct 10: 1235-1242, 2019.

This work is licensed under a Creative Commons Attribution-NonCommercial-NoDerivatives 4.0 International (CC BY-NC-ND 4.0) License. 